\title{
Philosophiques
}

\section{Jocelyn Benoist, Kant et les limites de la synthèse. Le sujet sensible, Paris, PUF (Collection « Epiméthée »), 1996, 345 p.}

\section{Claude Piché}

Volume 24, numéro 2, automne 1997

URI : https://id.erudit.org/iderudit/027466ar

DOI : https://doi.org/10.7202/027466ar

Aller au sommaire du numéro

Éditeur(s)

Société de philosophie du Québec

ISSN

0316-2923 (imprimé)

1492-1391 (numérique)

Découvrir la revue

Citer ce compte rendu

Piché, C. (1997). Compte rendu de [Jocelyn Benoist, Kant et les limites de la synthèse. Le sujet sensible, Paris, PUF (Collection " Epiméthée »), 1996, 345 p.] Philosophiques, 24(2), 442-444. https://doi.org/10.7202/027466ar d'utilisation que vous pouvez consulter en ligne.

https://apropos.erudit.org/fr/usagers/politique-dutilisation/ 
Jocelyn Benoist, Kant et les limites de la synthèse. Le sujet sensible, Paris, PUF (Collection « Épiméthée »), 1996, 345 p.

Pour mener cette recherche sur la subjectivité, Jocelyn Benoist adopte la méthode phénoménologique, bien qu'il ne vise pas, à la manière de Husserl, à privilégier une sphère de données absolues sous la forme, par exemple, d'un ego transcendantal (p. 167), mais qu'il parte plutôt de la philosophie critique de Kant, cet a inventeur " de la subjectivité (selon le mot de Fichte [p. 11]), afin de montrer dans quelle mesure la transcendance est déjà inscrite au coeur de la subjectivité kantienne. Le sujet connaissant est d'emblée a dehors ", il se transcende vers le monde comme en témoigne la constitution de l'expérience objective. Parmi les conditions qui rendent possible cette expérience, l'auteur choisit de porter son attention de manière privilégiée sur la sensibilité - d'où le sous-titre de l'ouvrage, Le sujet sensible - , ce qui l'amènera en fin de parcours à élaborer une áidétique complète de la sensation •(p. 240). On le voit, l'ouvrage cherche à réhabiliter à sa manière l'Esthétique transcendantale en affrontant de plain-pied les problèmes qu'elle pose.

La Logique transcendantale, même s'il y est abondamment question de la subjectivité, ne parvient en définitive qu'à un résultat négatif à cet égard. D'une part, la déduction des catégories fait intervenir un sujet transcendantal qui, parce qu'il est condition de possibilité de toute objectivité, se soustrait irrémédiablement au statut d'objet (p. 89). D'autre part, lorsque la psychologie rationnelle, prise à partie dans le chapitre traitant des Paralogismes, cherche à voir dans le sujet pensant une substance, c'est-à-dire à le ramener au statut d'objet, elle succombe au leurre de l'apparence transcendantale ( $\mathrm{p}$. 135) et se voit contrainte de céder la place à une très précaire psychologie empirique. Or, si ce bilan de la Dialectique transcendantale ne peut être considéré comme le dernier mot de Kant à propos de la subjectivité, il constitue néanmoins pour Benoist une invitation à revenir en arrière et à envisager sous un jour nouveau le sens interne, tel qu'il est introduit dans l'Esthétique transcendantale .

L'auteur, il va sans dire, n'est pas le premier à vouloir mettre en relief la finitude du sujet kantien dans sa dimension de réceptivité. Bien avant lui, Heidegger avait déjà, sur fond d'analytique existentiale, mis en lumière la supériorité du temps sur l'espace, annoncée dans l'Esthétique, en vue de montrer ultérieurement le caractère central des schèmes transcendantaux, à titre de règles de détermination du temps. Mais l'auteur rétorque qu'il n'existe aucune raison de privilégier ainsi de manière unilatérale le temps aux dépens de l'espace puisque, comme l'énonce la seconde édition de la 
Critique, l'espace est “ l'horizon indépassable de l'“ intuitivité " conme telle v (p. 207), ce en quoi il détient une supériorité sur le temps. En d'autres termes, il est la condition indispensable de toute présentation sensible, de la mise en image de tout concept. Dès lors, si l'espace compte au nombre des " conditions extrinsèques du schématisme „, il n'en représente pas moins une condition essentielle au procès d'objectivation. Mais la critique du privilège insigne accordé au temps par Heidegger a une portée beaucoup plus profonde ici. En effet, le temps saisi comme "autoaffection * conduit Heidegger à une conception de l'ipséité qui répète, à son insu, le geste de l'idéalisme, en ce qu'une subjectivité ainsi repliée sur elle-même ne peut avoir qu'une " sensibilité sans sensation ` (p. 260), sans altérité véritable, sans contenu. La facticité du Dasein investie dans le sujet kantien est en vérité une "facticité sans fait ", sans le "fait de l'événement sensible v (p. 15).

Pour aborder l'étude de la sensation, essentielle à son projet d'une phénoménologie de la subjectivité sensible, l'auteur s'inspire plutôt du néo-kanticn Hermann Cohen. D'abord, Cohen souligne avec force qu'à elle seule la sensation ne peut garantir à l'objet son existence ; au contraire, la réalité objective du phénomène ne peut être attestée que lorsque la sensation vient s'inscrire clans une synthèse catégoriale (p. 234). Ensuite, et ceci vient compléter les développements de l'Esthétique, la sensation ne peut à proprement parler "affecter" "le sujet que si elle procède de l'objet et donc, si l'affection a est elle-même catégorialement déterminée " (p. 270). L'auteur reconnaît ainsi à Cohen le mérite d'avoir mis en lumière ces aspects de la sensation qui, loin d'être adéquatement traités dans l'Esthétique transcendantale, relèvent en fait de la Logique transcendantale. En revanche, le phénomène de la sensation ne peut être entièrement absorbé par l'Analytique, sous peine de clonner prise à une nouvelle forme d'idéalisme absolu (p. 239). La contribution de l'Analyrique transcendantale au problème de la sensation (et du sens interne) se limite donc à ce que la " synthèse transcendantale n est en mesure d'expliciter, d'où le titre de l'ouvrage Les limites de la synthèse. La synthèse catégoriale n'en représente pas le fin mot, tant s'en faut. Et cette mise au point vaut tout autant pour Cohen, qui absolutise l'Analytique des principes au point d'y intégrer l'Esthétique, que pour Heidegger, qui surinvestit l'imagination productrice à l'œuvre dans le schématisme (p. 175, 255).

Le sujet doit donc être saisi en cleçà des synthèses dont il est l'auteur. C'est-à-dire qu'il doit lui-même faire l'objet d'une " thèse $n$ : il est posé au sens où il se trouve, en vertu de sa sensibilité conçue comme " passivité originaire ", " exposé " (p. 15-16). Or, seul un réexamen de la thématique de l'affection, centrale pour la sensation, permet de montrer comment le sujet se découvre ainsi a transi d'extériorité ". Et ce, non pas au sens où, déjà constitué comme sujet autonome, il serait interpellé par une quelconque instance extérieure, mais bien plutôt en ce que le moi ne peut advenir à lui-même indépendamment de l'affection, qui le révèle pour la première fois à lui-même. " Nous sommes affectés " par" des objets et cette affection nous fait apparaître à nous-mêmes comme étant affectés "(p. 276). Avant même qu'il n'exécute de synthèses cognitives, le sujet, parce qu'il est en son fond même asensible ", se doit d'advenir à lui-même malgré - ou plutôt grâce à - un “ désœuvrement n originaire. Suivant cette piste, riche d'enseignements, jusque dans la troisième Critique, Benoist parvient à dégager la signification profonde de la finitude comme passivité première, par delà l'ontologie heideggérienne du Dasein. Il dévoile ainsi une dimension encore négligée de la sensibilité chez Kant, dimension à laquelle quiconque s'intéresse à la théorie de la subjectivité sera désormais tenu de se confronter, pour son plus grand profit. 
Les limites de la synthèse est un ouvrage fort bien documenté et rédigé dans un style à la fois rigoureux et spéculatif. On regrettera seulement que l'auteur, après avoir nettement marqué son refus du a réalisme de la chose en soi * (p. 264), ne revienne plus par la suite sur ce thème, pourtant indissociablement lié au problème de la sensation. Il y va en effet de la dimension de contingence propre à la a donation .

Claude Piché

Département de philosophie

Université de Montréal 